\title{
Variation und Wandel der Vokale im Lateinischen
}

\author{
Carlotta Viti
}

\section{Einleitung}

Der für das Urindogermanische rekonstruierte Vokalismus wird im Lateinischen gut bewahrt; so werden hierbei die zwei Quantitäten (lang vs. kurz) und die fünf Qualitäten $(a, e, i, o, u)$ des Urindogermanischen erhalten, und zwar ähnlich wie im Altgriechischen, im klassischen Armenisch und in den keltischen Sprachen. Dagegen vermischen andere idg. Sprachen und Sprachfamilien die Qualität besonders der mittleren und niedrigen Vokale: ${ }^{*} a$ und ${ }^{*} o$ werden in den slawischen, baltischen und germanischen Familien durcheinander gebracht (natürlich nicht auf dieselbe Weise), und sogar noch größer ist diese Verwirrung im Indo-Iranischen, wo ${ }^{*} a$ und ${ }^{*} o$ nicht nur miteinander, sondern auch mit $*_{e}$ vermischt werden.

Aber hinter dieser treuen Bewahrung zeigt das Latein wichtige Neuerungen und Schwierigkeiten, sicher mehr als das Altgriechische. Der altgriechische Vokalismus ist nicht von Akzent, Silbenstellung oder Öffnung bedingt, während solche Faktoren eine große Rolle in dem lateinischen Vokalinventar spielen. Das bewirkt von Haus aus mehr Variation in den Vokalen des Lateinischen als in denen des Altgriechischen. Wie Bartoněk bemerkt, "wenn man über die Geschichte der phonologischen Analyse der lautlichen Subsysteme beider klassischen Sprachen, des Altgriechischen und des Lateinischen, nachzudenken versucht, muss man gleich am Anfang zur Kenntnis nehmen, dass die Bedingungen für eine gründliche Analyse der phonemischen Subsysteme an der griechischen Seite unvergleichbar günstiger sind. "(1996: 117) 
Die Schwierigkeiten des lateinischen Vokalismus hängen teilweise davon ab, dass die Natur des Akzentes, der die Vokale des Lateinischen so stark bedingt, nicht so gut bekannt ist. Die Forscher behaupten, dass dieser Akzent in vorgeschichtlichen Zeiten anders als der Akzent war, der vom Klassischen Latein, Altgriechischen und Vedischen belegt wird, und der für das Urindogermanische rekonstruiert wird; der urlateinische Akzent war wahrscheinlich nicht melodisch, sondern intensiv wie der Akzent der romanischen Sprachen, und besonders intensiv auf der ersten Silbe, wie der Akzent der alten keltischen und germanischen Sprachen (vgl. Baldi 2002: 268ff). Die spezielle Rolle, die die erste Silbe des Wortes im Keltischen und im Germanischen spielt, erscheint in dem gewöhnlichen Gebrauch der Alliteration in der Wurzel der Wörter, und dasselbe geschieht in der Dichtung oder dichterischen Prosa des Altlateinischen.

Es soll bemerkt werden, dass der lateinische Vokalismus vor allem aus der Perspektive des Lautwandels beschrieben wurde, den die Junggrammatiker festgestellt haben, d.h. als ein blindes und ausnahmsloses Phänomen (\$2). Das gibt aber manchmal den unangenehmen Eindruck, dass es sich um eine lange Serie getrennter Lautgesetze handelt, die die wichtige Generalisierung nicht erfassen, dass ähnliche Phänomene auch in anderen Sprachen mit einer gewissen Ordnung vorkommen. Weitere Perspektiven des Lautwandels wurden in jüngerer Zeit vorgeschlagen, vom Strukturalismus (sowohl à la Saussure als auch à la Chomsky, vgl. §3) und vom Funktionalismus, der oft die Ansätze der Soziolinguistik und der Psycholinguistik ins Feld führt (vgl. §4). Solche Modelle waren bei vielen Erklärungen des Lautwandels erfolgreich, aber sie werden selten auf die Phonologie des Lateinischen oder der anderen alten idg. Sprachen angewendet, die normalerweise die Festung des junggrammatischen Denkens sind. In diesem Artikel werden wir sehen, wie einige dieser nachjunggrammatischen Ideen des Lautwandels helfen können, um den Vokalismus des Lateinischen besser zu verstehen. Obwohl es nicht völlig klar erscheinen wird (was fast unmöglich für eine tote Sprache ist), können sie es vielleicht ein bisschen weniger schwer machen. Aber zuerst ist es notwendig, die traditionellen Begriffe des lateinischen Vokalismus in Betracht zu ziehen. 


\section{Phonetische Regularität}

Die junggrammatische Perspektive über Lautwandel liegt mehr oder weniger explizit den meisten Beschreibungen des lateinischen Vokalismus zugrunde (vgl. Juret 1921: 248ff; Meillet-Vendryes 1979: 108ff; Leumann 1977: 43ff; Sommer \& Pfister 1977: 39ff; Sihler 1995: 35ff; Meiser 1998: 55ff; Baldi 2002: 242ff; Clackson \& Horrocks 2007: 92ff, usw).

Als Erstes wird von den Grammatiken gesagt, dass lange Vokale stabiler als kurze Vokale sind; das ist offensichtlich in der Entwicklung nicht nur von dem rekonstruierten Urindogermanischen zum Lateinischen $\left({ }^{*}\right.$ mäter $>$ māter 'Mutter', *rëgs > rēx 'König', *vīvos > vīvus 'lebend', *dõnom > dōnum 'Gabe', *dhümos > fümus 'Rauch'), sondern auch vom Lateinischen zu den romanischen Sprachen, die normalerweise die Qualität der langen Vokale des Lateinischen bewahren (vgl. die italienischen Entsprechungen madre, re, vivo, dono, fumo). Dagegen erleben kurze Vokale mehrere Veränderungen. Dasselbe geschieht in anderen Gruppen der Indogermania; z.B. sind im Slawischen die ultra-kurzen Vokale, die jers genannt werden, sehr früh verschwunden.

Zweitens sind kurze Vokale in der ersten Silbe stabiler als in anderen Stellungen des Wortes - das ist eine Darstellung der Tatsache, dass der Akzent ursprïnglich auf der ersten Silbe war. Am Anfang bewahren oft die lateinischen kurzen Vokale die idg. Qualität, wie in ăgō vs. Grie. ã $\gamma \omega$, fẽrō vs. Grie. $\phi \epsilon ́ p \omega$,

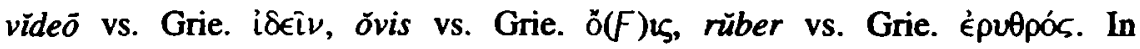
Mittestellung zeigen aber kurze Vokale Phänomene von Schwächung, und in Endstellung werden sie oft verloren.

Drittens ist der Lautwandel der inneren kurzen Vokale weniger dramatisch, wenn sie in einer geschlossenen Silbe sind, als in offenen Silben - die Schließung der Silbe ist ein Schutz für die vokalische Klangfarbe. Z.B. leitet die Vokalschwächung vom inneren $\check{a}$ zu $\check{e}$ in einer geschlossenen Silbe (facio vs. confectus) aber zu $\check{r}$ in einer offenen Silbe (facio vs. conficio). Natürlich ist der niedrige Vokal $a$ von einem artikulatorischen Standpunkt aus dem mittleren Vokal $e$ näher als dem hohen Vokal $i$.

In diesem Rahmen wirken die phonetischen Gesetze ausnahmslos, z.B. wird idg. * ${ }^{\check{e}} \mathrm{zu}$ Lat. $\check{o}$ vor einem labialen Approximant oder einer velaren Liquida ( $l$ pinguis), wie in (1). 


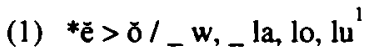

*newos > novus 'neu', Grie. vé(F)os

*velō > volō 'ich will', vgl. velle

Wenn ein Lautgesetz einige Gegenbeispiele hat, kann es davon abhängen, dass ein spezifischeres Gesetz angewendet wird, oder dass eine Darstellung der Analogie vorkommt. Während Analogie auch die Morphologie betrifft, bezeichnet ein spezifischeres Gesetz ein weiteres Phänomen, das völlig phonetisch bedingt wird, und das deshalb auch unter dem Begriff der Ausnahmslosigkeit eingeschlossen werden kann. Normalerweise wird das spezifischere Gesetz früher als das generellere Gesetz angewendet, das so in seiner Domäne beschränkt wird. Z.B. wird das obere Gesetz in (1) nicht angewendet, wenn der Vokal $\check{e}$ nach einem palatalisierten Konsonant $c$ oder $\boldsymbol{g}$ steht: *gelu > gelu 'Frost', Ita. gelo. SchlieBlich ist dieses Phänomen für die Tatsache verantwortlich, dass die romanischen Sprachen Laute wie Italienisch [t] [\$] oder Französisch [ $s$ ] in diesen Kontexten belegen.

Die Hypothese der Ausnahmslosigkeit des Lautwandels, die die Junggrammatiker in dem XIX. Jahrhundert eingeführt haben, wird heutzutage auch als im Wesentlichen korrekt berïcksichtigt. Z.B. sammelt The Handbook of Historical Linguistics herausgegeben von Joseph \& Janda (2003) Beiträge, die zu verschiedenen Forschungstraditionen gehören, vom Generativismus zum Funktionalismus, aber alle stimmen mehr oder weniger über die Haltbarkeit des junggrammatischen Modells überein. Janda (2003: 406), der keinem spezifischen theoretischen Rahmen besonders folgt und immer sehr vorsichtig in der Analyse der Daten ist, behauptet, dass die Bedingungen des Lautwandels am Anfang immer phonetisch sind, und dass andere morphologische oder semantische Faktoren später eingreifen. Sogar die Kritik von Kiparsky (2003), der so viel zu der

\footnotetext{
'Diese Notation besteht aus zwei Teilen, d.h. dem so genannten 'strukturellen Wandel' (structural change) einerseits (in diesem Fall "wird $e$ zu $o$ ") und der so genannten "strukturellen Beschreibung' (structural description) andererseits (in diesem Fall "vor $w$ oder vor l pinguis"). Sie wurde eigentlich von der Generativgrammatik vorgeschlagen, seit der Zeit von The Sound Pattern of Englisch von Chomsky und Halle (1968), aber sie wird heute auch von Indogermanisten angenommen (vgl. Hock 1991: 26ff), weil sie erlaubt, alle Sprachwandel auf eine einfache Weise darzustellen.
} 
modernen phonologischen Theorie beigetragen hat, spricht nicht das Prinzip an, dass der Lautwandel ausnahmslos, blind, phonetisch bedingt sein und auf artikulatorischen Prozessen basieren kann; Kiparsky redet eher solche Indogermanisten an, die nur die Ausnahmslosigkeit der Lautgesetze berücksichtigen ("The existence of an important class of exceptionless sound changes grounded in natural articulatory processes is not in doubt, of course. It is the claim that it is the only kind of sound change that is under question", 2003: 313). Nach Kiparsky ist der wichtigste Einwand gegen das junggrammatische Modell des Lautwandels die so genannte 'Struktur-Dependenz' (structure dependence), d.h. die Tatsache, dass das phonetische Inventar einer Sprache immer irgendeine Konsequenz hat. Wir werden die Struktur-Dependenz des lateinischen Vokalinventars in der folgenden Sektion diskutieren.

\section{Struktur-Dependenz}

\subsection{Kohärenz des lateinischen Vokalinventars}

Nach dem Strukturalismus (vgl. Jakobson 1929) und der Generativgrammatik (vgl. Chomsky \& Halle 1968; Kiparsky 2003) können Lautwandel nicht völlig blind sein, sonst hätten die Sprachen nach Jahrtausenden vom Lautwandel riesige phonetische Inventare, die jeder Analyse bezüglich Kohärenz und Ordnung widerstehen. Ganz im Gegenteil folgen Lautwandel immer bestimmten phonologischen Prinzipien, die sprachübergreifende ziemlich ähnliche Phoneminventare schaffen. Z.B. haben alle Sprachen normalerweise Verschlusslaute, und unbedingte Lautwandel vom Streben zu Frikativlaut bewirken keine Leerstellen in dem Verschlusslautsystem.

Eine konsequente innere Organisation kann auch im Lateinischen erscheinen. Erstens betreffen die Lautwandel dieser Sprache meistens die vorderen Vokale

\footnotetext{
${ }^{2}$ Nach der Generativgrammatik sind die zugrunde liegenden phonologischen Prinzipien solcher Systeme angeboren. Funktionalisten und Typologen verpflichten sich nicht zum Angeborensein solcher Prinzipien - tatsächlich ist der Nativismus eine nicht so interessante Sache für sie, da er nicht dargelegt und deshalb nicht widerlegt werden kann - aber sie sind im Großen und Ganzen mit der Behauptung einverstanden, dass die Phoneminventare der verschiedenen Sprachen eine gewisse Kohärenz haben, die auch durch implikationale Universalien à la Greenberg beschrieben werden können.
} 
einerseits $(e>i$ oder $i>e$ ) und die hinteren Vokale andererseits ( $o>u$ oder $u>$ $o$ ), während Kreuz-Wandel normalerweise vermieden werden. Besonders die mittleren Vokale $e$ und $o$ haben oft eine parallele Entwicklung; im Bezug auf den Lautwandel von $i \mathrm{zu} e$ und von $u \mathrm{zu} o$ vor einem $r$, das von $s$ stammt, bemerkt Baldi (2002: 246), dass "the changes of $i>e$ and $u>o$ are parallel in that both involve the lowering of a high to a mid vowel in a phonologically identical environment". Cf. (2) und (3).

(2) *si-s-ö > serō 'ich säe', vgl. sēuī

(3) *fusēd > forēt 'esset', vgl. Osk. fusid

Zweitens drücken die drei Vokale $a i u$ am Rand des Dreieckes die Qualitäten aus, die mehr den verschiedenen Lautwandeln widerstehen. Was z.B. kurze Vokale in der ersten Silbe betrifft (vgl. Meillet \& Vendryes 1979: 111), wechselt ein $\breve{a}$ am Anfang überhaupt nicht. Die anderen Vokale ändern sich zwar, aber die Änderungen des mittleren Vokals $\check{e}$ sind zahlreicher als die Änderungen des hohen Vokals $\check{i}$, d.h. $\check{e}$ ist instabiler als $\check{i}$. Tatsächlich ist der Wandel vor einem $r$, das von s stammt, wie in (2) der einzige regelmäBige Fall, wo ein $\check{l}$ ändert. Dagegen kann ein $\check{e}$ nicht nur vor einem Approximant und vor einem $l$ pinguis wie in (1), wo das Ergebnis $\check{o}$ ist, sondern auch vor einem velaren Nasal, wie in (4); in diesem Fall ist das Ergebnis ein $\check{l}$.

(4) $\check{e}>\grave{1} / \ldots$

${ }^{*}$ tengō $>$ ting $(u) \bar{o}$ 'ich benetze', vgl. Grie. TÉ $\gamma \gamma \omega$

Ähnlich sind die Änderungen des mittleren Vokals $\check{o}$ zahlreicher als die Änderungen des hohen Vokals $\breve{u}$. Ein $\breve{u}$ kann nur vor einem $r$ ändern, das von $s$ stammt, wie in (3), aber ein $\check{o}$ ändert in den folgenden Kontexten: vor einer Liquida plus Konsonant (5a); vor einem Nasal (5b) - in beiden Fällen ist das Ergebnis $\check{u}$; vor einem labialen Approximant (6) - hier wird das $\check{u}$ zu $\check{a}$; in Sequenzen wie wor, wos, wot $(7)$ - hier wird das $\check{o} \mathrm{zu} \check{e}$.

(5) $\check{\delta}>\breve{\mathrm{u}} /$ _ $1+$ Konsonant, _ + Nasal

(5a) volt $>$ vult 'er will', vgl. volumus

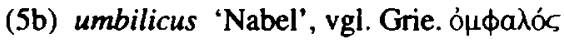

(6) $\zeta>a / \_w$

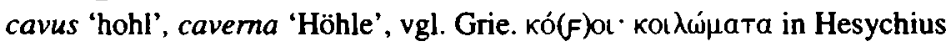

(7) $\check{o}>\check{e} / w_{-} r, w_{-} s, w_{-} t$ vortō > vertō 'ich wende', vgl. divortium 
Wie man sehen kann, ist $o$ instabil, sicher instabiler als $u$. Die größere Stabilität der Vokale $a i \boldsymbol{u}$ an der Peripherie des vokalischen Dreieckes im Vergleich mit der der mittleren Vokale $e o$ ist ein klarer Beweis der Tatsache, dass die Vokale des Lateinischen nach einer 'Abwärtsstrukturierung' (top-down structuring) organisiert sind, die die Lautwandel auf die Dauer beschützen. Denn wie die phonetische Forschung zeigte, stellen die drei Vokale $a \boldsymbol{i} u$ die extremen Punkte in dem verfügbaren vokalischen Raum dar, und können sehr einfach voneinander unterschieden werden (Lindblom 1986; Bybee 2001). Deshalb sind $a i u$ auch die häufigsten Vokale in den Phoneminventaren der Sprachen der Welt (Maddieson 1984: 123ff). Wenn eine Sprache nur drei Vokale hat, sind diese $a i u$; das geschieht z.B. im Arabischen.

Dieselbe Kohärenz ist in Diphthongen offensichtlich. Das Urindogermanisch erlaubte eine große Flexibilität in der Kombination der verschiedenen Vokale in einem Diphthong; z.B. hatte es sowohl vordere Vokale mit vorderen Halbvokalen (ei) als auch vordere Vokale mit hinteren Halbvokalen (eu). In allen idg. Sprachen werden solche kombinatorischen Möglichkeiten mit der Zeit reduziert, und das geschieht natïrlich auch im Lateinischen, aber mit konsequenten Entwicklungen, nach denen 'homogene' Diphthonge wie ei viel besser als 'dishomogene' Diphthonge wie eu bewahrt werden. ${ }^{3}$ Im Allgemeinen ist eu der Diphthong, der im Altlateinischen am wenigstens bewahrt ist: er ist schon in vorgeschichtlicher Zeit verschwunden, und besonders ist er als ou in den ersten Belegen geschrieben. In der Grabinschrift von L. Cornelius Scipio Barbatus, die auf das 3. Jahrhundert v.Chr. zurückgeht, ${ }^{4}$ stellt die Form abdoucit eine Wurzel *deuk dar, die mit der Schwundstufe *dŭk in Ablaut ist (vgl. DELL 185). Vgl. (8).

(8) subigit omne Loucanam opsidesque abdoucit

"Er unterwarf die ganze Lukanien und zog die Geisel ab" (CIL 12 7)

${ }^{3}$ Die gute Bewahrung der Diphthonge au und ai (später in der Form ae) ist kein Gegenbeispiel, weil a zentral ist, und deshalb bildet es keine unhomogene Kombination mit den Halbvokalen.

${ }^{4}$ Die Möglichkeit, dass die Elogia Scipionum eine spätere Version der Kaiserzeit sind, spielt hier keine große Rolle, da die Schrift jedenfalls archaisierend ist, in Übereinstimmung mit den echten Urkunden des Frühlateinischen. Vgl. die Diskussion in Baldi (2002: 206ff); Clackson \& Horrocks (2007: 138ff). 
In (8) kommt der Diphthong ou auch in dem Nomen Loucanam 'Lukanien' vor; auch hier geht er auf den ursprünglichen Diphthong *ei zurück, wie in den

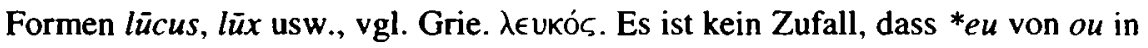
abdoucit und Loucanam vertreten wird: im Gegensatz zu eu ist ou homogen, denn es besteht aus einem hinteren Vokal und einem hinteren Halbvokal. Andererseits wird $e i$ bis zu den Urkunden des II. Jahrhunderts oft geschrieben und von $i$ unterschieden; in dem Senatus Consultum de Bacchanalibus (186 v. Chr.) kommt ei oft vor (quei, foederatei, exdeicendum, usw.), vgl. (9). In dem Senatus Consultum gibt es keinen Beleg von eu.

(9) sei ques esent quei sibei deicerent necesus ese bacanal habere, eeis utei ad pr(aetorem) urbanum Romam venirent

"Sollte es Personen geben, die erklären, (einen Platz für) ein Bacchanal nötig zu haben, müssen sie zum Stadtprätor nach Rom kommen" (CIL $1^{2}$ 581)

\subsection{Asymmetrie in dem lateinischen Vokalismus}

Die Kohärenz des Vokalinventars des Lateinischen bedeutet zwar eine ratio aber nicht notwendigerweise Parallelismus oder Symmetrie. Ganz im Gegenteil zeigen die Vokale einer Sprache oft Leerstellen und asymmetrische Verteilungen. Im Allgemeinen erscheinen vordere Vokale unmarkiert, weil sie synchronisch häufiger und diachronisch besser im Lautwandel bewahrt sind. In der Synchronie, wenn eine Sprache ein kleines Vokalinventar hat (d.h. weniger als 5 Vokale, vgl. Maddieson 2005a), fehlen hintere Vokale häufiger als vordere Vokale (Ladefodeg 2005: 175). Das wurde von der Typologie festgestellt, aber wir können Beispiele auch in den alten idg. finden, wo z.B. das Hethitische keinen Unterschied zwischen $o$ und $u$ zeigt, während $e$ und $i$ normalerweise unterschieden werden. In der Diachronie bewies Labov (1994), dass die Richtung der Vokalwandel von hinteren zu vorderen Vokalen ist; ein bemerkenswertes Beispiel ist das Great Vowel Shift des Englischen, wie die Entwicklung

\footnotetext{
'In Fällen wie Lat. seu 'oder wenn' (<*sey-we), ceu 'wie' (<*key-we) usw. ist eu immer sekundär und stellt nicht den Diphthong eu des Urindogermanischen dar (vgl. Sihler 1995: 57).
} 
von hinteren gerundeten Vokalen zu vorderen gerundeten Vokalen im Deutschen, im Französischen und auch im Klassischen Griechischen.

Im Bezug auf das lateinische Vokalsystem erscheint dieser Prinzip klar, denn die Lautwandel, die die vorderen Vokale $e$ und $i$ betreffen, sind regulärer als die Lautwandel, die von den hinteren Vokalen $o$ und $u$ erlebt werden; ähnlich sind die Lautwandel, die zu den vorderen Vokalen $e$ und $i$ leiten, zahlreicher als die Lautwandel, die zu den hinteren Vokalen $o$ und $u$ leiten. Das kann besonders in den Entwicklungen der kurzen Vokale bemerkt werden, die weniger stabil als die langen Vokale sind.

In der ersten Silbe kann sich der mittlere und vordere Vokal $\check{e}$ entweder zu dem mittleren Vokal $\check{o}$ (z.B. *newm > novem, vgl. Grie. évvé( )a) wie in (1) oder zu dem vorderen Vokal $i$ (z.B. *dek-no- > dignus, vgl. decet) wie in (4) entwickeln. Aber die Ergebnisse von $\check{o}$ sind heterogener und weniger vorhersehbar: Meillet \& Vendryes (1979: 112) sagen, dass der Wandel von $\check{o}$ zu $\check{u}$ vor Velarnasal "sans règle bien claire" ist, und im Allgemeinen kann der mittlere hintere Vokal $\check{o}$ nicht nur zu dem hinteren Vokal $\breve{u}$ (z.B. uncus neben Grie.

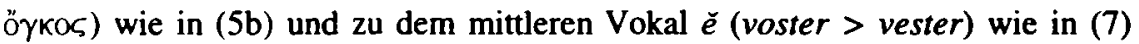

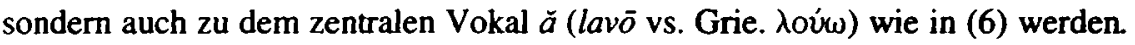
Der letzte Fall ist ziemlich willkürlich und wird von Meiller \& Vendryes als eine Darstellung von Dissimilierung beschrieben ähnlich der, wo $\bar{o}>\bar{a}$ vor dem Halbvokal $w$, vgl. octävus vs. octō (1948: 110). Selbstverständlich ist dieser ein sehr ungewöhnlicher Lautwandel im Lateinischen, das die ursprünglichen langen Vokale sehr treu bewahrt. Im Allgemeinen ist die Dissimilierung von Historikern der Sprache als ein sporadisches und unvorhersehbares Phänomen betrachtet, ungleich der Assimilierung (vgl. unten).

Dasselbe erschient in Mittestellung, wo die Vokalschwächung bewirkt. Hier kann das Ergebnis im Prinzip sowohl $\check{~}$ (facio vs. conficio) als auch $\breve{u}$ (taberna vs. contubernālis) sein. Aber de facto ist der Lautwandel der lateinischen Vokalschwächung zu dem vorderen Vokal $\check{l}$ viel häufiger als der zu dem hinteren Vokal $\check{u}$; denn der letzte ist normalerweise auf den Mechanismus der Ableitung beschränkt (mons vs. promunturium), während der erste produktiv in der Flexion ist (cadō vs. cecidī), und Flexion ist regelmäßiger als Ableitung. Baldi 
merkt richtig an, dass "the rule of vowel weakening [...] is actually a rule affecting non-back vowels" (2002: 253).

Diese Tendenz zu vorderen Vokalen kann auch einige schwierige Fälle der lateinischen Vokalschwächung erklären. Die Grammatiken berichten mehrere Schwankungen besonders vor Labialen: "bald $i$, bald $u$ (ohne daB es bisher gelungen wäre, die Gründe genau festzustellen, welche für die Entstehung der einen oder der anderen Klangfarbe maßgebend waren) vor den Lippenlauten $b$, $p, f, m$ " (Niedermann 1953: 23). Das ist der so-genannte sonus medius (Quint. Inst. Or. 1.4.8), der in Formen wie in (10) erscheint.

(10) aurifex vs. aurufex 'Goldschmied', intimus vs. intumus 'innerster', libet vs. luber 'es gefällt', mancipis vs. mancupis 'des Aufkäufers', optimus vs. optumus 'bester'

Baldis Meinung nach sind solche Paaren "without obvious patterns", so dass "it is better to see the fluctuation in these vowels as reflecting inconsistent orthography and regionally or socially determined variation" (2002: 254). Aber es ist auffällig, dass alle Beispiele der Grammatiken Wörter betreffen, wo ein hinterer Vokal $u$ phonetisch erwartet wird und stattdessen ein vorderer Vokal $i$ vorkommt: ein Labial sollte im Prinzip ein $u$ triggern, da $u$ ein gerundeter Vokal ist, und Rundung setzt eine Bewegung der Lippen voraus. Tatsächlich ist die Form mit $u$ oft alter als die entsprechende Form mit $i$ (z.B. "mancupis archaischer und mancipis klassischer Gen" von manceps, Niedermann 1953: 27). Nie geschieht das Gegenteil, nie haben wir eine Schwankung im Fall eines Wortes, wo $i$ vorkommen sollte. ${ }^{6}$ Deshalb können die unerwarteten Belege des vorderen Vokals $i$ als eine weitere Darstellung der Unmarkiertheit der vorderen Vokale interpretiert werden. Diese Asymmetrie bleibt aber unerklärt, wenn man das Modell der Junggrammatiker stricto sensu übernimmt.

\footnotetext{
${ }^{6}$ Ein ähnliches Phänomen involviert die Liquida: die palatale Liquida triggert zwar ein $i$, während ein $u$ eine Beziehung mit der velaren Liquida hat, z.B. Siculus vs. Sicilia, famulus vs. familia, exul vs. exilium usw. Aber es gibt auch hier Ausnahmen: aquila 'Adler', mutilus 'verstümmelt', nubilus 'wolkig', rutilus 'rötlich'. Nach Meillet \& Vendryes (1979: 113) sind diese Formen extreme Fälle des 'flottement' der Vokale in velaren Kontexten. Auch in diesem Fall $u$ ist der phonetisch erwartete Vokal, da $u$ ein hinterer oder velarer Vokal ist - hier wurde das $u$ aber ersetzt wegen einer Dissimilation mit den vorgehenden Vokalen (mutilus, nubilus, rutilus) oder Labiovelaren (aquila).
} 
Die Verbreitung der vorderen Vokale erscheint auch in Diphthongen. Wir haben oben gesehen, dass homogene Diphthonge wie ei und ou besser als dishomogene Diphthonge wie $e u$ und oi bewahrt sind. Aber zwischen den zwei homogenen Diphthongen $e i$ und $o u$, ist der erste überwiegend gewöhnlicher in den alten Urkunden. In dem Senatus Consultum de Bacchanalibus z.B. kommen 39 Belege von $e i$, hier aufgeführt in (11), und 7 Belege von $o u$, hier in (12).

(11) quei (3 Mal), foederatei, exdeicendum, neiquis, sei (3 Mal), sibei, deicerent, eeis (2 Mal), utei (10 Mal), ubei, ceivis, nisei (3 Mal), preivatod, oinuorsei, virei (2 Mal), interibei, exdeicatis, eis, inceideretis, seiqua, ibei, vobeis.

(12) iousiset, coniouras, plous (3 Mal), noundinum, ioubeatis.

In dem ganzen Senatus Consultum de Bacchanalibus gibt es 53 Diphthonge, deshalb kommt $e i$ in $73.6 \%$ der Fälle vor. ${ }^{7}$ Auch in diesem Fall ist die Häufigkeit ein klares Zeichen der Unmarkiertheit. Eine Bestätigung kommt von dem Altgriechischen, das am Ende des Wortes die idg. Diphthonge mit langem Vokal bewahrt, nur wenn sie einen Halbvokal ı einschließen, vgl. - ōı (Dat.Sg. der $\bar{\alpha}$ Stämme), - $\omega l$ (Dat.Sg. der o-Stämme), - $\eta \iota$ (Konj. 3Sg. der thematischen Verben), usw. Die idg. langen Diphthonge mit Halbvokal $v$ sind verschwunden.

Unter diesen Umständen müssen wir aber die Gründe dieser Unmarkiertheit untersuchen. Denn das Etikett 'unmarkiert' ist nur die Beschreibung - keine echte Erklärung - eines Phänomens; Erklärung setzt eine Beziehung mit etwas anderem voraus. In diesem Fall kann eine mögliche Erklärung der Unmarkiertheit der vorderen Vokale und Diphthonge im Lateinischen in einigen artikulatorischen Fakten anderer Laute gefunden werden, und besonders in den Koronalen. DefinitionsgemäB ist die 'Korona' (der Zungensaum oder Zungenkranz) das Artikulationsorgan der Koronalen wie $n, l, r, s, z, t, d, t s, d z$. Es gibt eine große empirische Evidenz der Unmarkiertheit der Koronalen. Erstens sind Koronalen normalerweise häufiger als andere Konsonanten in dem Phoneminventar einer Sprache: wenn eine Plosive fehlt, dann ist das der Labial $p$ zwischen Stimmlosen und der Velar $g$ zwischen Stimmbaften, wie im Etruskischen; die

\footnotetext{
${ }^{7}$ Die Belege von ai sind duelonai, haice, aiquom, tabelai, datai (in aedem ist ai schon $\mathrm{zu}$ ae geworden). Die Belege von oi sind: comoine, oinusorgei.
} 
Dentale $t$ oder $d$ fehlen normalerweise nicht (Maddieson 2005b). Es gibt mehr Typen von Koronalen (Dentalen, Alveolaren, Alveopalatalen, Retroflexen) als Typen von Labialen oder Velaren; nach einem implikationalen Universal setzt die Anwesenheit eines Labialnasals die Anwesenheit eines Dentalnasals voraus. aber nicht umgekehrt (Ferguson 1966); ähnlich kommen Koronalfrikativen wie Spiranten häufiger als andere Frikativen mit einem labialen oder velaren Artikulationsort vor (Maddieson 1984: 126). Zweitens überwiegen Koronalen in vielen phonologischen Prozessen andere Konsonanten, wie in Dissimilation (Grie. à $v-\delta$ - $\rho$ ós vs. áví $\rho$ ), Lösung von Konsonant-Gruppen (Lat. factu(m) > Ita. fatto) oder Neutralisierung. Z.B. erlaubt das Altgriechische nur die Koronalen [ $n$ ] [ $r$ ] [ s ] am Ende eines Wortes, und besonders zeigt es eine Neutralisierung der Nasale: der Dentalnasal [ $\mathrm{n}$ ] überwiegt den Labialnasal [ $\mathrm{m}$ ]:

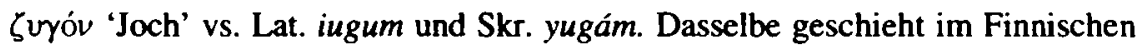
(Blust 1979; Houlihan \& Iverson 1979: 56-57). Deshalb können Koronalen als phonologisch unterspezifiziert betrachtet werden. Drittens werden Koronalen besonders frühzeitig von Kinder während des Spracherwerbs benutzt (Kean 1980). Im Allgemeinen stimmen die meisten Forscher in the special status of coronals (Paradis \& Prunet 1991) überein.

Wahrscheinlich besteht eine Beziehung zwischen der Tatsache, dass der Labialkonsonant $p$ und der Velarkonsonant $g$ sprachübergreifend seltener als die entsprechenden Dentalkonsonanten sind, und der Tatsache, dass der hintere Vokal $u$ weniger häufig in dem Vokalinventar einer Sprache ist. In beiden Fällen wird ein vorderer Laut (ohne Bewegung der Lippen) einem hinteren Laut bevorzugt. Das hat eine artikulatorische Motivierung, die von einem funktionellen Standpunkt aus normalerweise einleuchtender als eine akustische Motivierung ist. Wie Martinet (1955) bemerkt, gibt es zwei gegenüberstellende Funktionen im Lautbildungsprozess, d.h. Ökonomie und Deutlichkeit: nach der Ökonomie neigt der Sprecher zu einem schnellen Ausdruck, und das ergibt phonologische Prozesse wie Lösung, Schwächung, Assimilation usw.; nach der Deutlichkeit soll der Hörer die linguistische Mitteilung gut verstehen, und das ergibt phonologische Prozesse wie Gemination, Fortition, Dissimilation usw. Die Lautwandel der Lösung, Schwächung und Assimilation sind sprachübergreifend häufiger und regelmäßiger als die Lautwandel der Gemination, For- 
tition und Dissimilation - es gibt z.B. wenige Sprachen wie das Lateinische oder das Italienische, wo Geminaten einen phonologischen Charakter haben - und Dissimilation wird als ein 'sporadisches' Phänomen in Büchern über Lautwandel betrachtet (Hock 1991: 107ff). Das weist darauf hin, dass der Sprechakt kein symmetrischer Akt ist, da die Funktionen des Sprechers stärker als die Funktionen des Hörers sind. In unserem Fall gibt es kein Grund von einem akustischen Standpunkt aus, wofür ein vorderer Laut einem hinteren Laut bevorzugt wird; es wurde nicht dargelegt, dass vordere Laute einfacher zu unterscheiden oder wahrzunehmen sind. Aber von einem artikulatorischen Standpunkt aus ist dieser Grund offensichtlich. Denn die Zunge bleibt in ihrer naturellen Richtung in der Aussprache der vorderen Laute, während die hinteren Laute eine Bewegung rückwärts der Zunge voraussetzen (und die Seltenheit der Labiale im Vergleich mit Dentalen hat etwas mit der Tatsache zu tun, dass die Zunge viel mehr Möglichkeiten in der Konstriktion als die Lippen hat).

\section{Lexikalische Diffusion und andere semantische Faktoren}

Nach den oberen Bemerkungen kann der lateinische Vokalismus in einem umfassenden Rahmen aufgefunden werden, im Bezug auf artikulatorische Fakten und auf die Verteilung der Vokale in anderen Sprachen. Aber natürlich kann der lateinische Vokalismus nicht völlig auf diese Weise erklärt werden, da es immer eine wesentliche Anzahl von Ausnahmen und Idiosynkrasien gibt. Ein Modell, das oft hilfreich für die Erklärung der Ausnahmen eines Lautwandels erschien, ist die so genannte 'lexikalische Diffusion' oder die 'lexikalische Konnexion'.

Die lexikalische Diffusionshypothese stammt von der Dialektologie und der Soziolinguistik, seit der Zeit von Jaberg (1908) und Gilliéron (1912), nach denen jedes Wort seine eigene Geschichte hat. Sie wurde besonders auf den Lautwandel von Wang (1969), Chen \& Wang (1975), Labov (1994) usw. angewendet. In seinen zahlreichen Studien des in Philadelphia gesprochenen Englischs hat Labov gezeigt, dass viele UnregelmäBigkeiten von der Tatsache motiviert werden können, dass der Lautwandel - und im Allgemeinen der Sprachwandel - nicht homogen in der Gesellschaft fortgeht, sondern dass er sich nach Schichten, Alter, Geschlecht usw. ändert. Der Sprachwandel ist nicht 
abrupt: es gibt immer langsamere und schnellere Darstellungen einer Aussprache (leading and lagging pronounciations), und eine solche synchronische Variation in der Gesellschaft bewirkt den Lautwandel in der Diachronie. Obwohl nicht jede Variation in der Synchronie zum Lautwandel leitet, gibt es keinen Lautwandel ohne synchronische Variation. Deshalb, um einen Lautwandel zu verstehen, muss man die Variation durchschauen, besonders wie sich ein bestimmter Lautwandel durch das Lexikon verbreitet.

Die Anwendung der lexikalischen Diffusion auf eine tote Sprache ist sicher schwierig und in einigen Fällen umstritten, weil wir oft die genaue Bedeutung eines Wortes nicht völlig begreifen können. Hier beschränken wir uns auf drei Fälle, wo der Lautwandel der lateinischen Vokale nicht strukturell bedingt ist, sondern von semantischen Faktoren beeinflusst wird.

Als Erstes erwähnen wir den Fall der anscheinend idiosynkratischen Bewahrung von diphthongierten Formen in oe (13) bis zur klassischen Zeit; manchmal belegt dasselbe semantische Feld sowohl diphthongierte als auch monophthongierte Formen nebeneinander, wie in (14). Vgl. Meillet \& Vendryes (1979: 117-18); Sihler (1995: 54).

(13) proelium 'Schlacht', foedus 'Vertrag', moenia 'Mauern'

(14) poena 'Strafe' vs. püniō 'strafen', Poenus 'Punier' vs. Pūnicus 'id.'

Für solche Beispiele ist eine lexikalische Diffusion wahrscheinlich, weil es sich um Wörter handelt, die einerseits einen ähnlichen phonetischen Kontext darstellen, d.h. der Diphthong kommt zwischen einem Labial und einem Dental, und die andererseits die offizielle Sprache des Rechtes betreffen, denn das gerichtliche Register ist oft konservativ. Eine ähnliche phonetische Darstellung und eine semantische Beziehung sind die gewöhnlichen Voraussetzungen eines Lautwandels, der sich durch lexikalische Diffusion verbreitet. Sihler (1995: 54) fechtet die Möglichkeit an, dass der Bewahrung der Diphthonge in diesen Wörtern eine ratio unterliegt: "the appeal to the special character of the affected vocabulary as an explanation for the retention of $o e$ is obviously shaky: in the case of *moyn- giving both moenia 'walls' and mūnia 'duties', the latter would 
seem to be the technical term, not the former". ${ }^{8}$ Aber da der Diphthong oe normalerweise mit der Zeit monophthongiert wird, müssen wir nicht Formen wie mūnia erklären, sondern nur Formen wie moenia, die gegen die Tendenz verstoßen. Außerdem setzt die lexikalische Diffusion keine bestimmte Bedeutung per se voraus, sondern eine Beziehung verschiedener Wörter miteinander, in diesem Fall müssen wir nicht den Kontrast zwischen moenia und mūnia erläutern, die semantisch nichts gemeinsam haben und nur homophon sind (vgl. DELL 409-10; 422), sondern eher die Beziehung zwischen moenia und der semantisch verwandten monophthongierten Form mürus. Obwohl beide Formen die Mauer einer Stadt bezeichnen können (anders als paries 'Hausmauer, Wand'), ist moenia das technische, offizielle Wort, während mürus mehr die Alltagssprache betrifft (vgl. DELL 409-10).

Als zweites erwähnen wir die anscheinend inkonsequenten verschiedenen Entwicklungen der Wörter aetas und novitas (15)-(16). "En face de grec neotēs, ancien *newotä( $(t)$, le latin a novitās, dérivé de novus; mais, en face de aevum, le dérivé *aiwo-tāt- qui doit se présenter sous la forme aevitas - et qui y aboutit en effet, car cette forme archaïque est conservée dans l'orthographe de la Loi des XII tables - aboutit à aetas, par suite de l'amuissement de la voyelle brève intérieure après $-w$ - et entre deux syllabes longues." (Meillet 1948: 134)

(15) novitas 'Neuheit' < * novo-tät

(16) aetas 'Alter' < *aevo-tãt

Auch in diesem Fall müssen wir an die paradigmatische Beziehung zwischen solchen Nomina und den anderen Wörtern desselben semantischen Felds denken. Das Adjektiv novus, von dem novitas abgeleitet wird, ist sehr häufig -

${ }^{8}$ Die Argumentation von Sihler setzt fort: "and foedus 'foul' and foeteō "have a bad smell' can hardly have been 'technical or literary'” (2002: 54). Zusätzlich zu der möglichen Erklärung einer euphemistischen Entstellung oder Tabus, die Sihler selbst erwähnt, kann man sich an die Beurteilung von Emout \& Meillet erinnern, nach der es eine Variation zwischen den Schriften foeteo und faeteo im Altlatenischen gibt, und die letzte scheint auch auf einem semantischen Standpunkt besser, da Formen mit dem Diphthong ae oft physikalische Mängel bezeichnen (aeger, caecus, taeter, paedor usw.; vgl. DELL 244). 
tatsächlich ist 'neu' ein der häufigsten Adjektiven in einer Sprache (vgl. Dixon 1977). Deshalb kann das Nebeneinander mit novus die Bewahrung des Vokals in novitas begünstigen, so dass die zwei Wörter formell ähnlich bleiben. Obwohl aevitas semantisch verwandt mit aevum ist, ist das Abstrakt aevum nicht so häufig, und daher kann es für den Sprecher weniger wichtig sein, die synchronische Transparenz zwischen diesen zwei Wörtern zu erhalten. Das erinnert an den Begriff der 'Voraktivierung' (priming), der von der Psychologie und Neurologie abgeleitet wird (vgl. Kandel et al. 2000: 1230), der aber heutzutage auf linguistische Phänomene, auch in der Phonologie (vgl. Kiparsky 2003: 328-29) angewendet wird. Die Versuchsperson erlebt mehrere Reize, und dann wird gebeten, bestimmte Urteile in einem Test zu geben. Z.B. hört eine Versuchsperson mehrere Wörter, die mit $t i$ - beginnen und wird dann gebeten, den Namen eines Möbelstücks zu sagen: sie wird wahrscheinlich Tisch sagen; stattdessen, wenn sie mehrere Wörter hört, die mit st- beginnen, wird die Versuchsperson wahrscheinlich Stuhl sagen. In unserem Fall kann ein Laut besser bewahrt werden, wenn er mit anderen besonders häufigen Wörtern eine semantische Beziehung hat. Neben der lexikalischen Diffusion und der linguistischen Voraktivierung kann auch ein dritter semantischer Faktor die Entwicklung der lateinischen Vokale erklären. Um das einzuleiten, ist es nützlich wieder die Wörter von Meillet zu zitieren:

Du reste, il est malaisé de voir pourquoi une même voyelle finale se maintient ici et disparaît là : ita 'ainsi' qui répond exactement à iti 'ainsi' du sanskrit a toujours $a$ final ; mais son corrélatif $u t$ n'a jamais $a$; et, si l'on n'avait la forme archaïque aliuta 'autrement' où $-a$ figure clairement, et les combinaisons uti-que (de *uta-que), utei (de *uta-î) d'où $u t \bar{i}$, on ne devinerait la présence ancienne d'une voyelle finale que par le maintien de la sourde $-t$ : on sait qu'une dentale indo-européenne, finale depuis la langue commune, est toujours représentée en latin par $-d$, jamais par $-t$. (Meillet $1948:$ 136)

Wir können aber bemerken, dass ita und ut zu zwei verschiedenen Kategorien gehören, denn ita ist ein Adverb, $u t$ ist eine Konjunktion, und deshalb besitzt das erste eine konkretere, vollere Bedeutung als die zweite. Es ist wahrscheinlich, dass der semantische Inhalt der Wörter auch einen Einfluss (obwohl keine mechanische Bedingung) auf deren phonetische Entwicklung hat; gewissermaßen neigt der Sprecher dazu, 'salientere' Wörter sorgfältiger zu 
artikulieren, d.h. Wörter, die ihre eigene Bedeutung gut bewahren, während Funktionswörter wegen ihrer Häufigkeit normalerweise das erste Opfer eines schnellen Gespräches sind. Selbstverständlich setzt eine sorgfältige Aussprache auch eine treuere Darstellung der ursprünglichen phonetischen Form voraus. So wie Adverbien bedeutungsvoller als Konjunktionen sind, haben Nomina ihre eigene konkrete, kontext-unabhängige Bedeutung mehr als Adjektive, und sie sind auch referentieller als Verben. Daher, wenn ein Nomen einerseits und ein Adjektiv oder ein Verb andererseits, die semantisch verwandt sind, ein verschiedenes Verhältnis in Bezug auf phonetische Schwächung haben, können wir erwarten, dass das Nomen seine vokalischen Segmente besser als das Adjektiv oder das Verb bewahrt. Und genau das geschieht. Es ist vielleicht kein Zufall, dass es in den oben erwähnten Paaren poena vs. püniō und moenia vs. mūniō das Nomen ist, das die völlige Artikulation des Diphthongs erhält. Dasselbe gilt für das Paar poenus vs. pünicus: das Nomen poenus bewahrt den Diphthong, das Adjektiv pünicus wird monophthongiert. Beide Wörter können zwar gelegentlich sowohl als Nomina als auch als Adjektive benutzt werden, wie es oft der Fall in den alten idg. Sprachen ist, und im Allgemeinen ist die Zugehörigkeit einer syntaktischen Kategorie oft keine aut-aut-Frage, sondern ein allmähliches Phänomen. Aber poenus ist ein eigentliches Nomen, und seine adjektivale Funktion ist später und sekundär (vgl. DELL 518). Dagegen ist pūnicus 'mehr Adjektiv', wie man in den Ausdrucksweisen bellum pünicum, fides pūnica, mālum pūnicum 'Granatapfel' sehen kann; in diesen Strukturen kann pünicus nicht von poenus ersetzt werden. Von diesem Standpunkt aus sollte der lateinische Vokalismus besser in den Formen erhalten werden, die eine referentielle Funktion haben.

\section{Schluss}

Die synchronische Variation und der diachronische Wandel der lateinischen Vokale setzen nicht nur einen Mechanismus von blinden Lautgesetzen voraus, sondern zeigen auch Darstellungen von einer inneren Struktur-Dependenz. Wir haben aber gesehen, dass die Anwesenheit rationeller Prinzipien hinter dem Verhältnis der lateinischen Vokale keinen Parallelismus oder Harmonie bedeutet, weil das Vokalinventar des Lateinischen oft asymmetrisch ist, sowohl in 
der Synchronie als auch in der Diachronie. Im Allgemeinen werden vordere Vokale und Diphthonge bevorzugt im Vergleich mit hinteren Vokalen und Diphthongen: $e i$ ist im Altlateinischen besser bewahrt als $e u$, oi oder ou. Das findet Parallelen in anderen Sprachen, sowohl in Vokalen als auch in Konsonanten, wo die Koronalen - die einen vorderen Artikulationsort als Velaren haben - von einem artikulatorischen Standpunkt aus unmarkiert sind.

Wir haben auch bemerkt, dass das lateinische Vokalismus von einigen semantischen Bedingungen beeinflusst wird, wie lexikalische Diffusion, linguistische Voraktivierung und Referentialität. Während lexikalische Diffusion und Voraktivierung in der phonologischen Forschungen verschiedener modernen Sprachen schon benutzt wurden (aber nicht so viel in der Erklärung der phonologischen Systeme der alten idg. Sprachen), ist das Prinzip der Referenzialität im Bezug auf den Lautwandel am meisten unexploriert. Das kann von der Tatsache hängen, dass die Bedeutung der Wörter für den Sprecher der modernen idg. Sprachen oft synchronisch opak ist, während im Lateinischen die semantische Beziehungen zwischen verschiedenen Wörtern derselben Wurzel klarer war. Transparenz oder Ikonizität ist ein Grund dafür, dass eine Form bewahrt wird. Dagegen leitet eine höhere Opazität oder Arbitrarität zur Ökonomie der Form.

\section{Literaturverzeichnis}

Baldi, Ph. (2002) The foundations of Latin, Berlin-New York, Mouton de Gruyter.

Bartonek, A. (1996) "Das lateinische Vokalsystem", in Bammesberger, A. \& F. Heberlein (Hgg.) Akten des VIII. internationalen Kolloquiums zur lateinischen Linguistik, Heidelberg, Winter, 117-124.

Blust, R. (1979) "Coronal-non coronal consonant clusters: new evidence for markedness", Lingua 47: 101-117.

Bybee, J. (2001) Phonology and language use, Cambridge, Cambridge University Press. Chen, M. \& W. Wang (1975) "Sound change: actuation and implementation", Language 51:255-81.

Chomsky, N. \& M. Halle (1968) The sounds patterns of English, New York, Harper \& Row.

Clackson, J. \& G. Horrocks (2007) The Blackwell History of the Latin language, Oxford, Blackwell. 
DELL = Ernout, A. \& A. Meillet (1959) ${ }^{4}$ Dictionnaire Étymologique de la Langue Latine, Paris, Klincksieck.

Dixon, R. (1977) "Where have all the adjectives gone?" Studies in Language 1: 19-80.

Ferguson, C. (1966) "Assumptions about nasals; a sample study in phonological universals", in Greenberg, J. (Hg.) Universals of Language, Cambridge (MA), MIT Press, 53-60.

Gilliéron, J. (1912) Études de géographie linguistique, Paris, Champion.

Haspelmath, M. \& M. Dryer \& D. Gil \& B. Comrie (Hgg.) The World Atlas of Language Structures, Oxford, Oxford University Press.

Hock, H. (1991 $)^{2}$ Principles of historical linguistics, Berlin-New York, Mouton de Gruyter.

Houlihan, K. \& G. Iverson (1979) "Functionally-constrained phonology", in D. Dinnsen (Hg.) Current approaches to phonological theory, Bloomington, Indiana University Press, 50-73.

Jaberg, K. (1908) Sprachgeographie: Beitrag zum Verständnis des "Atlas linguistique de la France", Aarau, Sauerländer.

Jakobson, R. (1929) "Remarques sur l'évolution phonologique du russe comparée à celle des autres langues slaves", Travaux du Cercle Linguistique de Prague 2, nachgedr. in Selected Writings, Volume 1, Phonological Studies, The Hague, Mouton, 7-116.

Janda, R. (2003) "Phonologization as the start of dephonetization - or, On sound change and its aftermath: of extension, generalization, lexicalization, and morphologization", in Joseph, B. \& R. Janda (Hgg.), 401-422.

Joseph, B. \& R. Janda (2003) (Hgg.) The Handbook of Historical Linguistics, Oxford, Blackwell.

Juret, A. (1921) Manuel de phonétique latine, Paris, Hachette.

Kandel, E., I. Kupfermann, S. Iversen (2000) "Learning and memory", in Kandel, E., J. Schwarz, T. Jessel (Hgg.), Principles of Neural Science, New York, McGraw-Hill, 1227-1246.

Kean, M. (1980) The theory of markedness in generative grammar, Bloomington, Indiana University Linguistics Club.

Kiparsky, P. (2003) "The phonological basis of sound change", in Joseph, B. \& R. Janda (Hgg.), 313-342.

Labov, W. (1994) Principles of linguistic change, Volume 1, Internal Factors, OxfordCambridge (MA), Blackwell.

Ladefoged, Peter $(2005)^{2}$ Vowels and consonants, Oxford, Blackwell.

Leumann, M. (1977) $)^{2}$ Lateinische Laut- und Formenlehre, München, Beck.

Lindblom, B. (1986) "Phonetic universals in vowel systems", in Ohala, J. \& J. Jaeger (Hgg.) Experimental phonology, Orlando, Academic Press, 13-44.

Maddieson, J. (1984) Patterns of sound, Cambridge, Cambridge University Press.

Maddieson, J. (2005a) "Vowel quality inventories", in Haspelmath, M. et al. (Hgg.), 1417.

Maddieson, J. (2005b) "Voicing and gaps in plosive systems", in Haspelmath, M. et al. (Hgg.), 26-29.

Martinet, A. (1955) Économie des changements phonétiques, traité de phonologie diachronique, Berne, Francke. 
Meillet, A. (1948) Esquisse d'une histoire de la langue latine, Paris, Klincksieck.

Meillet, A. \& J. Vendryes (1979) ${ }^{5}$ Traité de grammaire comparée des langues classiques, Paris, Champion.

Meiser, G. (1998) Historische Laut- und Formenlehre der lateinischen Sprache, Darmstadt, Wissenschaftliche Buchgesellschaft.

Niedermann, M. (1953) Historische Lautlehre des Lateinischen, Heidelberg, Winter.

Paradis, C. \& J.-F. Prunet (1991) (Hgg.) The special status of coronals: internal and external evidence, San Diego, Academic Press.

Sihler, A. (1995) A new comparative grammar of Greek and Latin, New York \& Oxford, Oxford University Press.

Sommer, F. \& R. Pfister (1977) ${ }^{4}$ Handbuch der lateinischen Laut- und Formenlehre, Heidelberg, Winter.

Wang, W. (1969) "Competing changes as cause of residue", Language 45: 9-25. 